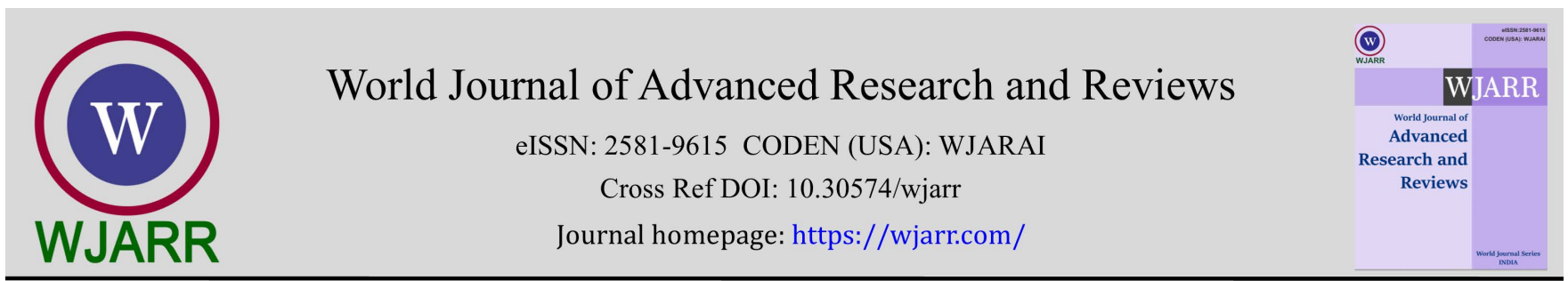

(RESEARCH ARTiClE)

\title{
The practical approach of Immuno histochemical markers in Solitary Fibrous Tumour (SFT)
} \author{
and Basavarajaiah Doddagangavadi Mariyappa 4,* \\ ${ }^{1}$ Department of Pathology, National Institute of Unani Medicine, Bangalore, Karnataka, India. \\ 2 Oncoville Cancer Hospital and Research Centre, Nagarbhavi, Bengaluru, India. \\ ${ }^{3}$ Department of Pediatrics, Bowring and Lady Curzon Hospital, Bengaluru, India. \\ ${ }^{4}$ Department of Statistics, Computer Science, KVAFSU (B), Hebbal, Bengaluru, India.
}

Renuka Bangalore Nagaraj 1, Anil Kumar Moodalbeedu Rajegowda 2, Murali Paramanandhan 3, Shivanna 2

World Journal of Advanced Research and Reviews, 2022, 13(02), 325-330

Publication history: Received on 23 December 2021; revised on 31 January 2022; accepted on 02 February 2022

Article DOI: https://doi.org/10.30574/wjarr.2022.13.2.0095

\begin{abstract}
A solitary fibrous tumour is an unknown neoplasm that is fibroblastic in nature. It causes diagnostic difficulty due to its unexpected Immunophenotypic appearance as well as wide histologic appearance, but most of the time it is accurately diagnosed due to its histological characteristics and strong positivity to $\mathrm{CD}_{34}$ expression.SFT is known to occur in almost all parts of the body including meninges and skin. This is a slow-growing neoplasm that can be successfully treated by complete excision; the malignant counterpart of SFT is rare. The most important and reliable positive markers in SFT are $\mathrm{CD}_{34}, \mathrm{CD}_{99}, \mathrm{BCL}_{2}$. There are consistently negative markers in this tumor as well, used according to the tumor location, such as EMA\&S $\mathrm{S}_{100}$.Although the majority of SFT can be accurately diagnosed due to histological characteristics and frequent $\mathrm{CD}_{34}$ expression, a wide histologic spectrum and the occurrence of an unexpected immunophenotype might offer diagnostic difficulties. Research findings shows that, the meninges, orbit, upper respiratory tract, salivary glands, thyroid, peritoneum, liver, retroperitoneum, pelvis, adrenal gland, kidney, urinary bladder, vagina, spinal cord, skin, periosteum, and soft tissues have all been known to have this form of neoplasm.
\end{abstract}

Keywords: Solitary fibrous tumour; IHC; Mesenchymal

\section{Introduction}

A solitary fibrous tumor (SFT) is a spindle cell mesenchymal tumor of poorly understood origin. In 1870 it was first diagnosed in pleura however its first description was made by Klemperer in 1931 where it was called localized fibrous mesothelioma. ${ }^{1,2}$ It was first mentioned in pleura in 1870 however the first description of this tumor was in 1931 by Klemperer, who called it "localized fibrous mesothelioma. 1,2 Hemangiopericytoma is now considered a cellular phenotypic variety of solitary fibrous tumour, which represents a single spectrum of mesenchymal malignancies. It is most common in adults and is thought to be of fibroblastic differentiation which can arise anywhere.3 SFT is made up of $\mathrm{CD}_{34}$-positive fibroblastic-looking cells that form a pattern less proliferation of alternating cellularity and collagenous stroma. While the majority of SFTs are benign and can be treated with surgery, 15-20 percent of people with SFT will develop local recurrence or distant metastases, which can be difficult to treat. It's uncertain whether SFTs that start in different places, including the meninges, lung, or breast, have the same pathophysiology 2,4. The liver, lungs, gastrointestinal tract, pancreas, adrenal, bladder, prostate, testis, thyroid, and salivary glands are all involved in rare cases. Cutaneous SFT appears to be uncommon, with only 20 cases recorded in the literature so far. Despite earlier studies showing an equal sex distribution of cutaneous SFT, investigations found a 4:1 male predominance. The skin of

\footnotetext{
${ }^{*}$ Corresponding author: Basavarajaiah DM

Department of Statistics, Computer Science, KVAFSU (B), Hebbal, Bengaluru, India.

Copyright (C) 2022 Author(s) retain the copyright of this article. This article is published under the terms of the Creative Commons Attribution Liscense 4.0.
} 
the head and neck appears to be the most typical location of genesis. ${ }^{5}$ Some SFTs were previously known as hemangiopericytomas, a name coined by Stout and Murray and publicized by Enzinger and Smith; however, this term has fallen out of favor, and SFT has largely superseded it. Hemangiopericytoma is no longer considered an obsolete synonym of SFT in the newest edition of the World Health Organization (WHO) classification of soft tissue and bone cancers. ${ }^{6}$ This tumour is most frequent in middle-aged adults (20-70 years old), with an average age of 44 years old. There have also been a few occurrences documented in the paediatric age categories. ${ }^{1,2,5}$ Although a small number of patients may suffer loco regional recurrence, SFTP is usually a benign, seldom aggressive malignancy. ${ }^{1}$ A malignant SFT can cause necrosis, bleeding, and wide attachment, as well as invading adjacent tissue, making total removal difficult 7,8

\section{Histopathology of SFT}

SFT has a wide range of histomorphology, ranging from paucicellular to moderately to highly cellular, with a round to spindle-shaped cells with little cytoplasm, between prominent eosinophilic bands of collagen, and is usually arranged in A storiform pattern along with hemangiopericytoma pattern. Often arranged in a short storiform pattern, and thinwalled branching vessels with a hemangiopericytoma-like configuration.

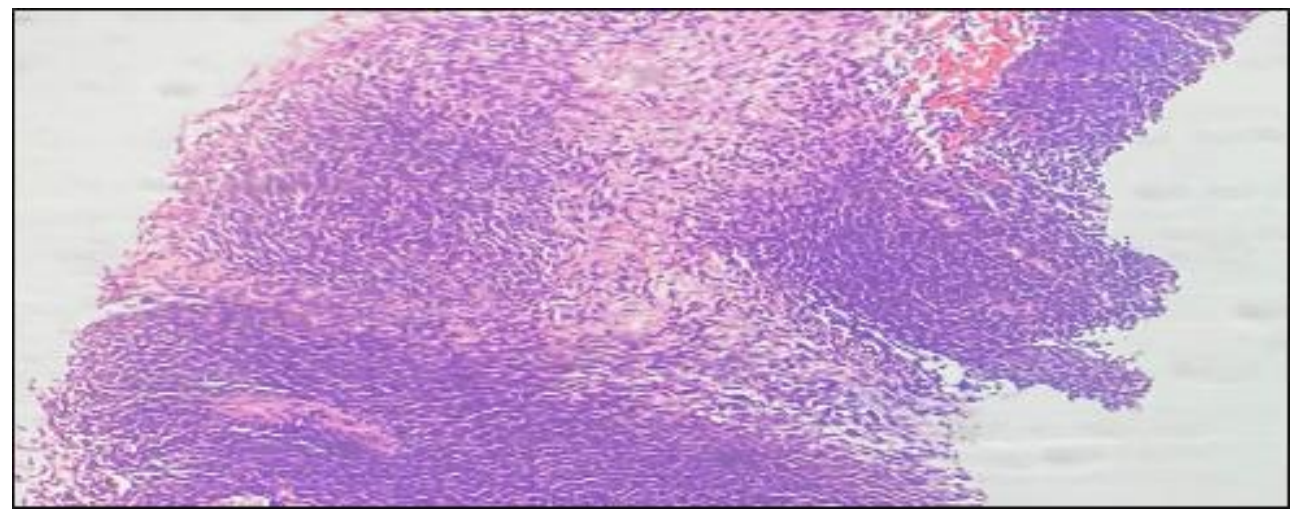

Figure 1 Histopathology of Tumour cells, hypo cellular and hypercellularareascan be seen

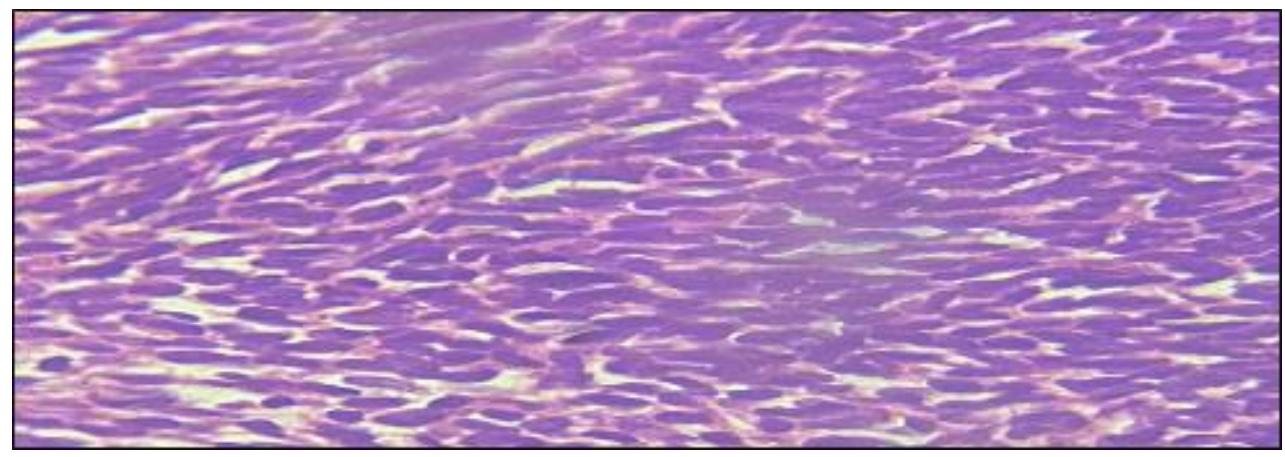

Figure 2 Hypo cellular areas was seen

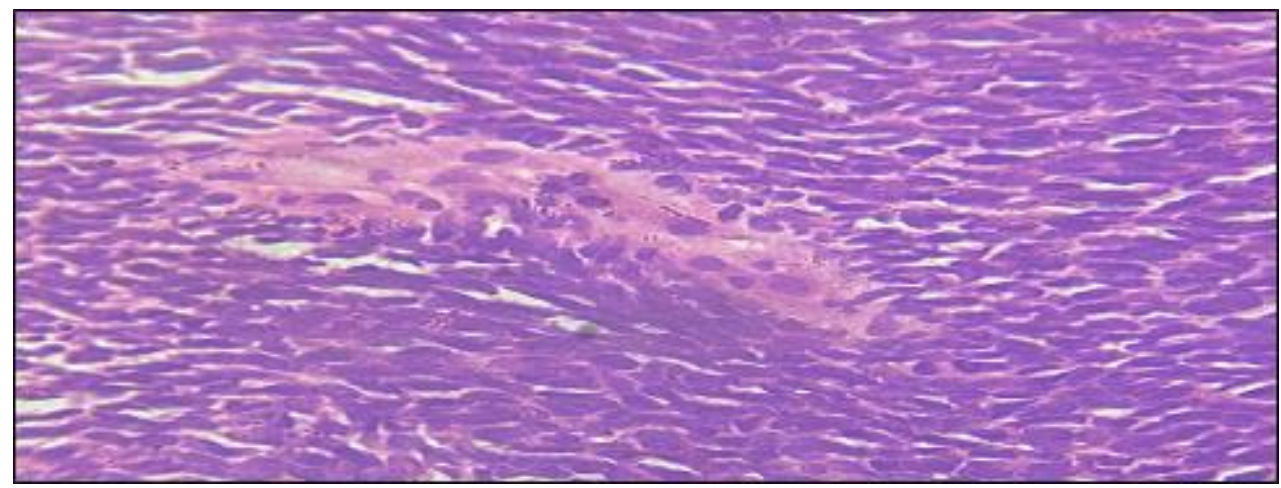

Figure 3 Tumour cells showing hemangiopericytoma like areas (HPE 40X) 


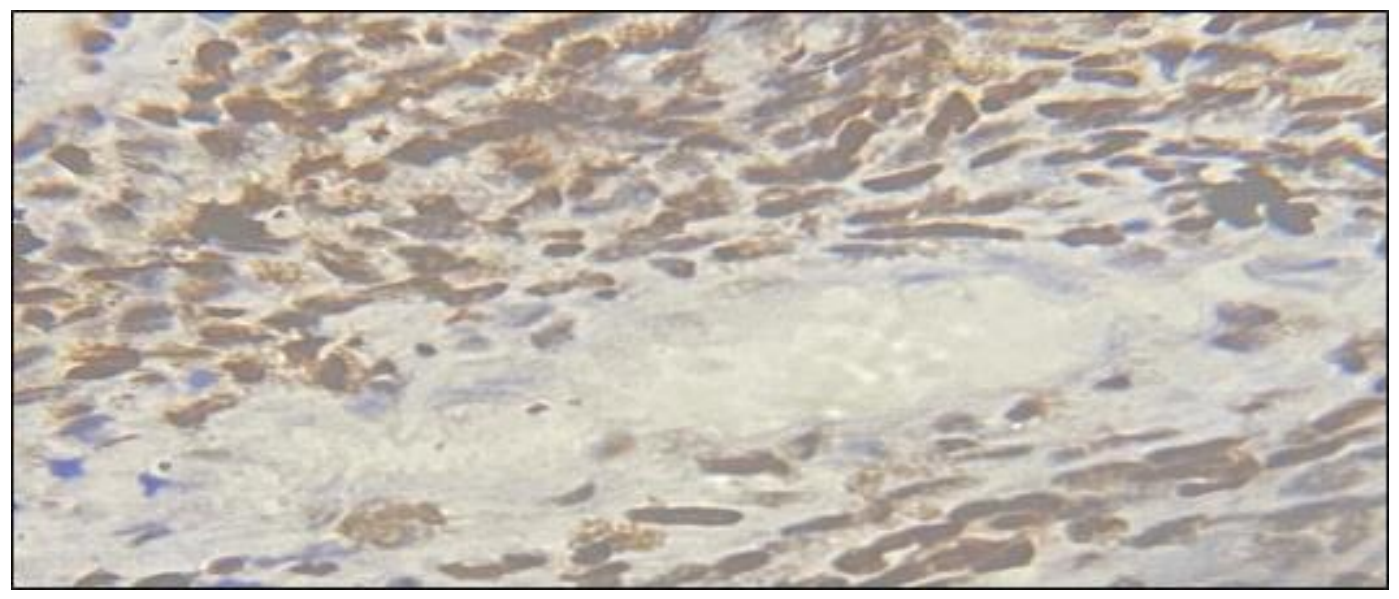

Figure 4 (IHC 40X) spindle cells positive for CD 34

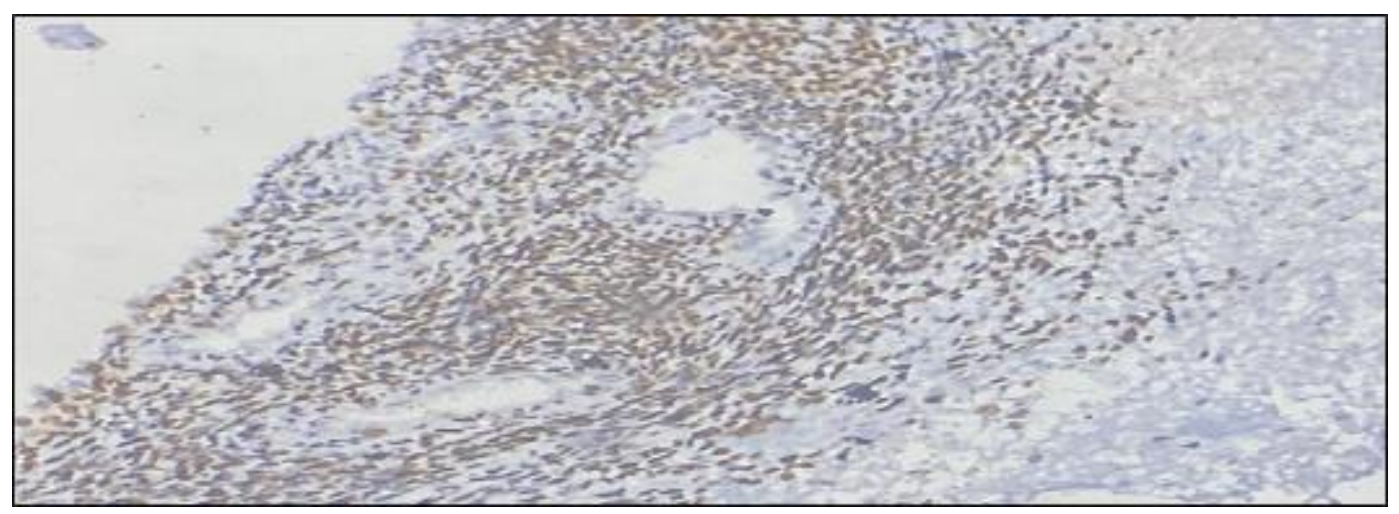

Figure 5 IHC 40 X-BCL2 spindles shaped cells showing positivity

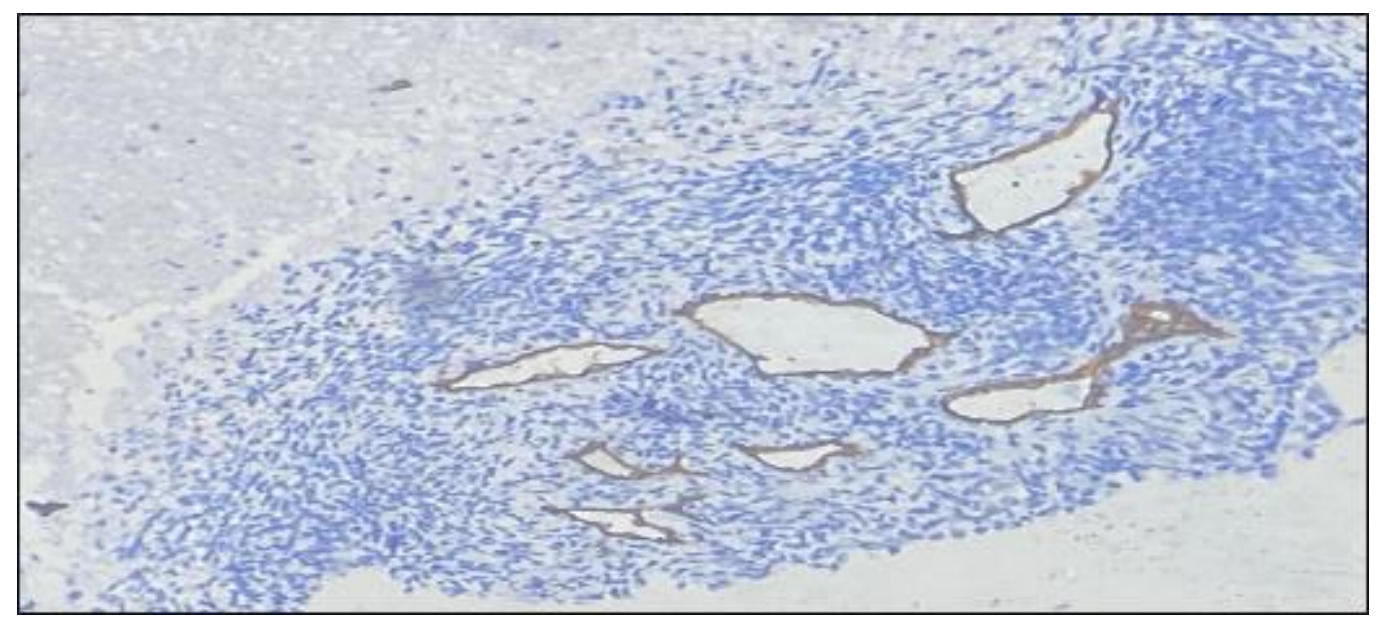

Figure 6 (IHC- S-100 40X) spindle cells showing negative marker for s-100

Soft tissue perineurioma, desmoidfibromatosis, spindle cell lipoma, and cellular angiofibroma are some of the benign soft tissue tumors that SFT may resemble histologically. Soft tissue tumors with the potential for locally aggressive behavior like desmoidfibromatosis and spindle cell sarcomas like malignant peripheral nerve sheath tumor, dermatofibrosarcomaprotuberans, and monophasic synovial sarcoma may be mimicked by SFT.8, 9 ,Small tumors with low mitotic rates are unlikely to metastasis, whereas giant tumors of $15 \mathrm{~cm}$ that arise in patients 55 years of age and with high mitotic figures are more likely to do so. Mitisisupto4/10 high-power fields necessitate close monitoring and are associated with a high risk of metastases and death. ${ }^{3}$ 


\section{Pleuroplumary SFT}

Stout and Murray described an intrapulmonary site for these tumors in 1942, although it appears that in these early descriptions, the tumour may have developed from the visceral pleura with an "inverted" growth pattern, suggesting an intrapulmonary lesion. ${ }^{10}$ These tumors are not related to asbestos or smoking, and they are more common between the fifth and eighth decades of life, with no sex predilection. ${ }^{11}{ }^{12}$, According to Keisuke et al ${ }^{2}$ out of the 21 tumors studied that had the $\mathrm{NAB}_{2}$ exon 4-Stat6exon two third fusion transcripts were pleuropulmonary SFTs. This was consistent with earlier findings, and showed that tumors with this fusion variation are associated with pleuropulmonary origin. ${ }^{13} \mathrm{CD}-$ 100 antibody staining, which is positive in SFTs and, according to some authors, represents a particular feature of these neoplasm, improves the histological evaluation of these tumours based on their immunohistochemical characteristics (keratin-negative, S-100-negative, vimentin-positive).,The completeness of the tumour resection determines the prognosis of patients with rare lung SFTs. SFTs of the lung that are malignant or suspected of being malignant should be treated as lung cancer in terms of surgical resection and follow-up. ${ }^{14}$ Caution is recommended, with close clinical follow-up for these tumours suggested regardless of histologic grade. ${ }^{10},{ }^{15}$ They are well-circumscribed, somewhat encapsulated, hard, white masses, typically greater than $10 \mathrm{~cm}$ in diameter, mainly associated to the visceral pleura, although they can also emerge from the parietal pleura or even within the lung in one third of the cases. ${ }^{7}$

\section{Abdominopelvic solitary fibrous tumours}

SFTs were formerly considered to only affect the pleura, however it is now known that they can occur in practically any area of the body, with ESFTs being more common than pleural SFTs. In 2012, Demicco et al., conducted a retrospective study of 110 cases of thoracic and extrathoracic SFTs, and found that the majority of cases were located in the abdominopelvic cavity (34\%). The pleura and extremities were less common primary sites(28\% and 16\%, respectively) and $(22 \%)$ of cases arose in the soft tissue of the head and neck (11\%) or trunk(11\%). ${ }^{3}$ Even in cases that have been recognized as benign, SFTs can develop late recurrences or metastases, according to the study, which looked at 10 cases of SFTs in the abdomen and pelvis that were retrospectively evaluated. As a result, extensive follow-up periods (up to 15 years) should be maintained, with a focus on the first two years. Wang et.al came to the conclusion that after surgical resection with free margins, the majority of abdominopelvic SFTs had a benign clinical history. Also they said that tumors that are larger than $10 \mathrm{~cm}$ or show a component of histological malignancy should be monitored more closely. ${ }^{15}$

\section{Solitary fibrous tumors (SFTs) in the head and neck}

SFT is a rare tumour in general, but it's very rare in the parotid gland. The exact cause of the tumour is unknown. While histogenesis has yet to be established, immunohistochemical and ultrastructural analyses have revealed that SFT is most likely formed from adult mesenchymal stem cells. Because SFTs are uncommon in the head and neck region, they must be considered in the differential diagnosis of head and neck spindle-cell malignancies. When combined with an awareness of the distinctive histopathologic aspects of SFT, CD 34 antigen expression is the most significant diagnostic tool. Positive margin is related to recurrence when the tumour is completely removed, with or without atypical or malignant microscopic findings. ${ }^{16}$

\subsection{Solitary fibrous tumour of the central nervous system}

Solitary fibrous tumours (SFT) of the central nervous system are uncommon neoplasms that manifest as dura-based masses and have a clinical appearance similar to meningiomas. ${ }^{2}$ Even in the meninges, where HPC has long been thought to be a separate tumour, there is some evidence that it belongs to the SFT spectrum and that lesions formerly labelled HPC are actually cellular forms of SFT. SFT has been described in one case that seemed extramedullary and mimicked meningioma. ${ }^{17}$

\subsection{Immunohistochemical positive markers for SFT}

Dan etal., found that all 7 benign and 11 malignant tumours had strong and diffuse Stat 6 immunstaining limited to the nucleus, which is a characteristic of solitary fibrous tumours. ${ }^{18}$. These data show that the reverse transcription polymerase reaction is the gold standard for detecting $\mathrm{NAB}_{2} / \mathrm{Stat}_{6}$ fusions across the spectrum, including dedifferentiated forms that have lost their immunohistochemical hallmark. ${ }^{4}{ }^{18}$. The molecular tests, on the other hand, are expensive and not available in every laboratory. Immunohistochemistry for Stat 6 has recently been introduced as a surrogate for identifying the fusion gene. A significant nuclear Stat6immunoreactivity was discovered, which was very sensitive and selective for SFTs. ${ }^{1}$ Leona et colleagues discovered that nuclear expression of Stat 6 was observed in 98 percent of a large cohort of SFTs (containing conventional, cellular, atypical, and malignant types). Stat 6 staining was often diffuse, with reactivity for Stat 6 in $>75$ percent of tumour cells in 68 percent of instances. Furthermore, staining 
intensity was strong in (67\%) of instances, moderate in 25\%, and weak in only (7\%). ${ }^{9}$ In a study by Gulsun Erdag et al, found that the most difficult differential diagnosis was Dermatofibrosarcoma, where Immuno profiles were similar findings and cytogenetic data argue against such a judgments between SFT and DFSP. Traditional karyotypic methods, fluorescence in situ hybridization, and the polymerase chain reaction all indicate translocations or ring chromosomes containing sections of chromosomes 17 and 22, however Dermatofibrosarcoma does not. SFT, on the other hand, does not have a consistent cytogenetic profile. Losses of portions of chromosomes 1, 3, 4, 8, 10,11,17, and gains of portions of chromosomes 1,2,3,4,6, 9, 13,18 have been documented in various analyses of the latter neoplasm. ${ }^{5}$., In contrast, substantial nuclear staining for $\mathrm{NAB}_{2}$ and Stat 6 was seen in all 40 samples. Strong nuclear expression of Stat 6 was also identified in one $\mathrm{CD}_{34}$-negative patient in a research by Keisuke et al. Stat 6 nuclear expression was found in both the conventional and dedifferentiated sections of two DSFT patients. ${ }^{13}$ Nicholas et al. found that malignant SFT has a worse prognosis than its benign counterpart. Surgical extirpation, in particular, has a definite role regardless of histologic subtype. Because present systemic therapy regimens do not appear to be beneficial, it is evident that patients with metastatic/unresectable illness require better systemic medications. ${ }^{19}$

\section{Conclusion}

The correct diagnosis of SFT is important as the management and prognosis are different as compared to its Malignant SFT. The distinct Immunohistochemical profile is not seen at all the time hence the diagnosis of SFT may be challenging at times. $\mathrm{NAB}_{2}-\mathrm{StaT}_{6}$ gene fusion is a sensitive and specific molecular marker that may be a helpful incorrect diagnosis. The management and prognosis of SFT differ from its malignant mimics and correct diagnosis is therefore important. Although SFT expresses a distinct Immunohistochemical (IHC) profile, the classic histomorphological and IHC profile is not seen in all cases and diagnosis can be challenging. $\mathrm{NAB}_{2}$-Stat 6 gene fusion has recently emerged as a sensitive and specific molecular marker and its IHC surrogate marker signal transducer and activator of transcription 6 (Stat6) has also been shown.

\section{Compliance with ethical standards}

\section{Acknowledgments}

The authors acknowledge the Director and all key officers of the National Institute of Unani Medicine, Bangalore, Karnataka, India for their kind support and cooperation in completion of the research work.

\section{Disclosure of conflict of interest}

There is no conflict of interest, this study is originally completed without any financial assistance from external agencies and parental Institutions.

\section{Statement of ethical approval}

The present study has carried out based on the observational approach, Institutional permission.

\section{Statement of informed consent}

Informed consent was obtained from all individual participants included in the study.

\section{References}

[1] Geramizadeh B, Marzban M, Churg A. Role of Immunohistochemistry in the Diagnosis of Solitary Fibrous Tumor a Review. Iran J Pathol. 2016; 11(3):195-203.

[2] Mekni A, Kourda J, Hammouda BK, Tangour M, Kchir N, Zitouna M, et al. Solitary fibrous tumour of the central nervous system : pathological study of eight cases and review of the literature. J Pathology. 2009;41(7):649-54.

[3] Demicco EG, Park MS, Araujo DM, Fox PS, Bassett RL, Pollock RE, et al. Solitary fibrous tumor: a clinicopathological study of 110 cases and proposed risk assessment model. Mod Pathol . 2012; 25(9):1298-306.

[4] Robinson DR, Wu Y-M, Kalyana-Sundaram S, Cao X, Lonigro RJ, Sung Y-S, et al. Identification of recurrent NAB2STAT6 gene fusions in solitary fibrous tumor by integrative sequencing. Nat Genet. 2013;45:180-7.

[5] Erdag G, Qureshi HS, Patterson JW, Wick MR. Solitary fibrous tumors of the skin: a clinicopathologic study of 10 cases and review of the literature. J Cutan Pathol. 2007; 34:844-50. 
[6] Yoshida A, Tsuta K, Ohno M, Yoshida M, Narita Y, Kawai A, et al. STAT6 Immunohistochemistry Is Helpful in the Diagnosis of Solitary Fibrous Tumors. Am J Surg Pathol. 2014;38:552-9.

[7] Karpathiou G, Stefanou D, Froudarakis ME. Pleural neoplastic pathology. Respir Med. 2015;1-13.

[8] Krishnamurthy S, Menon M, Parameswaran A, Sivaraman A. A case of solitary fibrous tumor of urinary bladder. Indian J Pathol Microbiol. 2021;64:847-9.

[9] Doyle LA, Vivero M, Fletcher CD, Mertens F. Nuclear expression of STAT6 distinguishes solitary fibrous tumor from histologic mimics. Mod Pathol. 2013;1-6.

[10] Rao N, Colby TV, Falconieri G, Cohen H, Moran CA, Suster S. Intrapulmonary Solitary Fibrous Tumors Clinicopathologic and Immunohistochemical Study of 24 Cases. Am J Surg Pathol. 2013;37:155-66.

[11] Arsalane A, Zidane A, Fenane H, Azami A, Essadi I, Raissi A, et al. Solitary Fibrous Tumor: Case Report of Intrapulmonary Location. Case Rep Oncol Med. 2018;1-4.

[12] Yoshida A, Tsuta K, Ohno M, Yoshida M, Narita Y, Kawai A, et al. STAT6 Immunohistochemistry Is Helpful in the Diagnosis of Solitary Fibrous Tumors. Am J Surg Pathol. 2014; 38: 552-559.

[13] Akaike K, Kurisaki-Arakawa A, Hara K, Suehara Y, Takagi T, Mitani K, et al. Distinct clinicopathological features of NAB2-STAT6 fusion gene variants in solitary fibrous tumor with emphasis on the acquisition of highly malignant potential. Hum Pathol. 2015;46: 347-56.

[14] Kouki HS, Koletsis EN, Zolota V, Apostolakis E, Dougenis D. Solitary fi brous tumor of the lung. Gen Thorac Cardiovasc Surg. 2008; 56: 249-51.

[15] Wang H, Chen P, Zhao W, Gu X, Xu Q. Clinicopathological findings in a case series of abdominopelvic solitary fibrous tumors. Onchology Lett. 2014;7:1067-72.

[16] Cox DP, Daniels T, Jordan RCK. Solitary fibrous tumor of the head and neck. Oral Surg Oral Med Oral Pathol Oral Radiol Endod. 2010;110:79-84.

[17] Mordani JP, Haq I, Singh J. Solitary fibrous tumour of the spinal cord. Neuroradiology. 2000;42:679-81.

[18] Dagrada GP, Spagnuolo RDVM, Tamborini E, Cesana L, Gronchi A, Stacchiotti S. Solitary fibrous tumors: loss of chimeric protein expression and genomic instability mark dedifferentiation. Mod Pathol. 2015; 1-10.

[19] DeVito N, Henderson E, Han G, Reed D, Bu MM, Lavey R, et al. Clinical Characteristics and Outcomes for Solitary Fibrous Tumor (SFT): A Single Center Experience. PLoS One. 2015;1-11. 